\title{
Theory of Social Returns in Portfolio Choice with Application to Microfinance
}

\section{Gregor Dorfleitner, Michaela Leidl and Johannes Reeder}

We complement standard portfolio theory à la Markowitz by adding a social dimension. We distinguish between two main setups, taking social returns as stochastic in the first, but as deterministic in the second. Two main features need to be introduced: Every asset must be assigned a (distribution of) social return(s), and the investor has to cherish social returns. The former comes with measurement problems, whereas the latter is mainly a problem of choice of a suitable utility representation. The focus of this paper is on the theoretical fundamentals and the practical implications of social returns. We apply each version of the theoretical model to a different realm. In the deterministic setup, we look at an investor who faces a small number of assets: the S\&P Euro Index, the EuroMTS Global Index, and the responsAbility Global Microfinance, where we assign a social return only to the microfinance investment fund. In the second application with stochastic social returns, we estimate statistical moments of social returns of various microfinance institutions and address the question how microfinance investment funds should allocate funds to microfinance institutions.

JEL Classifications: G11, G21, G32, D64, D81

\author{
CEB Working Paper No 10/014 \\ February 2010
}




\title{
Theory of Social Returns in Portfolio Choice with Application to Microfinance
}

\author{
Gregor Dorfleitner* $\quad$ Michaela Leidl $^{\dagger} \quad$ Johannes Reeder ${ }^{\ddagger}$ \\ February 21, 2010 \\ Preliminary Version
}

We complement standard portfolio theory à la Markowitz by adding a social dimension. We distinguish between two main setups, taking social returns as stochastic in the first, but as deterministic in the second. Two main features need to be introduced: Every asset must be assigned a (distribution of) social return(s), and the investor has to cherish social returns. The former comes with measurement problems, whereas the latter is mainly a problem of choice of a suitable utility representation. The focus of this paper is on the theoretical fundamentals and the practical implications of social returns. We apply each version of the theoretical model to a different realm. In the deterministic setup, we look at an investor who faces a small number of assets: the S\&P Euro Index, the EuroMTS Global Index, and the responsAbility Global Microfinance, where we assign a social return only to the microfinance investment fund. In the second application with stochastic social returns, we estimate statistical moments of social returns of various microfinance institutions and address the question how microfinance investment funds should allocate funds to microfinance institutions.

JEL-classification: G11, G21, G32, D64, D81

\footnotetext{
* University of Regensburg, gregor.dorfleitner@wiwi.uni-r.de

$\dagger$ University of Regensburg, michaela.leidl@wiwi.uni-r.de

† University of Regensburg, johannes.reeder@wiwi.uni-r.de
} 


\section{Contents}

1 Introduction 3

2 Theory 4

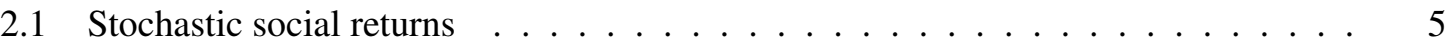

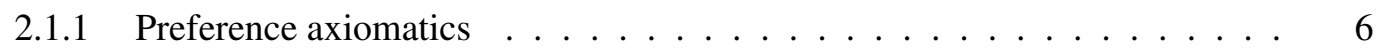

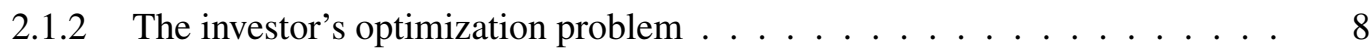

2.2 Deterministic social returns . . . . . . . . . . . . . . . . . . . 11

2.2.1 Axiomatics with deterministic social return . . . . . . . . . . . . . 11

2.2.2 Tobin-like separation . . . . . . . . . . . . . . . . . . . . . 12

3 Application $\quad 15$

3.1 Deterministic social returns: a socially-minded private investor . . . . . . . . . . 15

$3.1 .1 \quad$ Data . . . . . . . . . . . . . . . . . . 15

3.1 .2 Results . . . . . . . . . . . . . . . . . . . . 15

3.2 Stochastic social returns: microfinance . . . . . . . . . . . . . . . . . 18

$3.2 .1 \quad$ Data . . . . . . . . . . . . . . . . . . 18

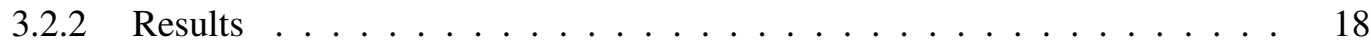

4 Research Outlook and Conclusion 23

5 Appendix 24

5.1 Expected utility vs. mean-variance . . . . . . . . . . . . . . . . . . . . . 24

5.2 Tobin-like separation: an alternative approach . . . . . . . . . . . . . . 25 


\section{Introduction}

The financial crisis has led many to question their investments. Apart from rethinking the risks involved, non-financial objectives have gained in importance. The terms 'Social Business' and 'Socially Responsible Investing' (SRI) are currently under discussion both in the general public and between researchers of various fields. ${ }^{1}$ Even though indisputable definitions are missing, the bottom line seems clear: Many people care about more than just financial returns. From a practical point of view, many of these people have too limited a circle of influence to actively engage in fostering social business. Apart from voluntary work in their leisure time, these people might express their preferences by taking into account the social dimension in their investment decisions. This tendency has brought up 'social funds', e.g., microfinance investment funds (MFIFs), renewable energy funds, and the like. The aim of these funds is to combine social and financial returns to attract investors.

The most common method to include a social dimension into investment choice is screening. ${ }^{2}$ The idea is simple: From all available assets, investors choose the subset of assets they want to invest in. 'Positive screening' picks out the assets to invest in, whereas 'negative screening' excludes assets which the investor does not want to fund under any circumstances. Screening usually takes place prior to any kind of optimization (if there is one). ${ }^{3}$ The optimization after screening could be done à la Markowitz, i.e., only depending on financial characteristics. In that case, once the subset of acceptable assets is chosen, there is no quantification of social returns. The combined procedure of screening and standard portfolio optimization might be considered a bounded rationality approach in that investors optimize only after having greatly reduced the complexity of the problem by having limited their choices available. Dupré, Girerd-Potin, and Kassoua (2004) apply screening to a large set of assets, determine the pre- and post-screening efficient frontiers and confirm intuition: By reduced diversification possibilities, the efficient portfolios after screening are financially worse than the ones before screening.

Clearly, social returns of individual assets differ considerably and might even be considered stochastic, as already noted by Dupré, Girerd-Potin, and Kassoua (2004). They mention two reasons for variations in social returns. The first concerns imperfect measurability of social returns. While being a genuine source of variation, this type of variation cannot be included into portfolio choice since it cannot be measured per se. Secondly, they point to variations of companies' social behavior over time, which we consider to be a valid reason for modeling social returns as stochastic.

The starting point for both the deterministic and the stochastic analysis is a metric scale to measure social returns. Once such a metric exists, empirical estimates of the mean and variance of social returns can be derived. Dependency structures between social returns of different assets can be estimated, as well as covariances between financial returns of one asset and social returns of

\footnotetext{
${ }^{1}$ A good starting point into the literature on SRI is the categorization of Hoepner (2007). The term 'social business' is strongly coined by Nobel Peace Prize winner Muhammad Yunus, e.g., see Yunus (2007). Yunus is also engaged in the yearly summit on social business organized by the Berlin-based Genisis institute.

2 For instance, see D’ Antonio, Johnsen, and Hutton (1997, 2000); Renneboog, Ter Horst, and Zhang (2008).

${ }^{3}$ Screening is not necessarily done before anything else. One could imagine a repeated optimization-screening pattern. After an initial optimization à la Markowitz, the optimal weights of assets could be checked and the assets (or amount of assets) incompatible with the investor's preferences could be sort out. The remaining assets could then be subject to another round of optimization.
} 
another. Apart from these inter asset dependencies, some investments have significant intra-asset covariances: A high financial performance goes at the expense of a low social performance and vice versa. Also, financial and social returns of an asset might depend positively on each other: A high financial performance is accompanied by a high social return.

Since uncertainty about financial returns can be accompanied by uncertainty about social returns, we consider stochastic social returns along with stochastic financial returns in our most general setup in Subsection 2.1. In Subsection 2.2, we reduce the analysis to deterministic social returns, for three reasons: First, we cannot get graphical results in the stochastic case since visual plots are restricted to be three-dimensional. Second, we work with real world data in Section 3. Estimates for variations in social returns are rather unreliable due to data restrictions. Third, we want to allow for the fact that, even if social returns are stochastic, people might not care about these variations. We further discuss this issue in Subsection 2.1. Deterministic optimization problems similar to our proposal have already been proposed and applied by Dupré, Girerd-Potin, and Kassoua (2004). However, they do neither present nor interpret any of the theoretical results we derive in Subsection 2.2. Furthermore, the efficient frontier they discuss is the standard two-dimensional financial meanvariance concept, although portfolios are assigned three dimensions. While we define, discuss and apply the concept of the efficient frontier in a five-dimensional space in the stochastic setup, we give a more intuitive discussion of the efficient frontier for deterministic social returns in threedimensional space together with a graphical exposition. In that respect, we want to mention Dunn (2006) who took an educated guess on the shape of a three-dimensional efficient frontier. We will confirm some general properties of his proposal in the deterministic setup, but add many others. After the theoretical exhibition, we use microfinance data to apply the model to two situations. First, in a setup with deterministic social returns, we look at an investor faced with a limited investment opportunity set, consisting of the Dow Jones Euro Stoxx Index, the EuroMTS Global Index, the responsAbility Global Microfinance Fund and a real estate index. Second, estimating social means, variances and covariances, we find optimal allocations for an MFIF which has to choose microfinance institutions (MFIs) to invest in.

\section{Theory}

As in standard portfolio theory, we consider a one-period investment problem, i.e., an investor at two points in time, where decisions are only taken at the first. At the second point in time, two types of returns accrue: financial returns $R$ and social returns $S$. Investors' preferences are formulated in terms of simple returns. This means that an investor allocating an initial wealth of $v_{0}$ gets a financial payoff of $v_{0} \cdot(1+R)$ plus a "social payoff" of $v_{0} \cdot S$ at the end of the period. Financial returns are measured in terms of money per unit invested, whereas the social return is a non-monetary value per unit invested. Therefore, both payoffs are not directly transferable into each other. The measurement of social returns is a problem in reality from which we abstain in the theoretical part in this section. Using rates of return instead of the absolute additional wealth and the additional social wealth implies that level-of-wealth effects have to be captured by the coefficients of the objective function to be defined further below. We assume that there is a metric for social returns which allows 
us to rank alternative investments in terms of social returns. This might be a (continuous or discrete) scale from some negative to some positive value, using zero as an average, e.g., for investments in a riskless asset. We could also use a non-negative support for social returns, having some positive number as the average.

With $N$ assets, each asset $i$ can be represented by a tuple of its financial and its social return, $\left(R_{i}, S_{i}\right)$. From all assets available, the investor builds a portfolio to maximize his objective function. Let $\mu_{R_{i}}$ be the expected financial return of asset $i, \sigma_{R_{i}}^{2}$ its variance and $\sigma_{R_{i}, R_{j}}$ the covariance between financial returns of assets $i$ and $j . R_{P}$ denotes the financial return of the portfolio and $S_{P}$ its social return.

$$
R_{P}=\sum_{i=1}^{N} x_{i} R_{i} \quad \text { and } \quad S_{P}=\sum_{i=1}^{N} x_{i} S_{i}
$$

where the vector $\vec{x}=\left(x_{1}, \ldots, x_{N}\right)^{T}$ contains the portfolio weights of the assets with $\sum_{i} x_{i}=1$.

\subsection{Stochastic social returns}

In the introduction, we have argued that, apart from inter-asset dependencies between financial returns, there might be significant inter-asset dependencies of social returns, as well as inter-asset dependencies of financial and social returns. We have also claimed that intra-asset dependencies between financial and social returns of an asset might be strong. A case in point for a negative intra-asset relationship can be found in the realm of microfinance. The idea of the trade-off is that microfinance borrowers ${ }^{4}$ are heterogeneous in quality so that high repayment rates and expected returns are more likely with less poor borrowers. Cull, Demirgüç-Kunt, and Morduch (2007) try to empirically verify the hypothesis of the trade-off and find some evidence in favor of it. ${ }^{5}$ In a recent study, Cull, Demirgüç-Kunt, and Morduch (2009, p. 182) write that "[d]ebate also persists on the extent to which trade-offs exist between pursuing profit and reaching the poorest customers. The data here suggest that this trade-off is very real".

One might object at this point that even though the trade-off exists, there need not be a stochastic element in it. If MFIs have different strategic orientations - some serving (local) middle-class borrowers, others the very poor - there might only be differences in financial returns and in (deterministic) social returns. However, there is another characteristic feature of the microfinance industry, namely that many MFIs depend on donations. ${ }^{6}$ This source of income is highly uncertain in the future and the financial uncertainty might also make social returns risky. Cull, Demirgüç-Kunt, and Morduch (2009, p. 182) speak of "fear that [...] institutions will sacrifice part of their social missions if subsidies are reduced sharply". An MFI which operates in a market environment and loses donor money might be forced to give up on some social returns to compensate for the loss of financial returns in order to stay attractive for MFIFs and other investors. ${ }^{7}$ In practice, this would be achieved by giving

\footnotetext{
${ }^{4}$ Here, we are talking about the borrowers who demand funds from MFIs, as opposed to the MFIs themselves which, among other sources, demand funds on capital markets.

${ }^{5}$ However, having disaggregated data, the authors are able to show that the strength and even the direction of the trade-off depends on institutional design and strategic orientation of the lender.

${ }^{6}$ In principle, the same logic applies to other firms in the realm of subsidy-receiving industries, as most renewable energy companies, for instance.

${ }^{7}$ This chain of arguments assumes the aforementioned trade-off between financial sustainability and outreach.
} 
larger loans to less poor people, neglecting the very poor. There are at least two positive effects on financial returns. First, the less poor (and supposedly more educated and productive) clientele might repay more often, and second, giving larger loans reduces transaction costs. The first channel is supported by Morduch (2000, p. 621) who asserts that "[p]roducing and selling goods requires more than just capital. It requires skills, other materials, information, connections, transportation, etc. Since richer households tend to have more of these inputs, marginal returns to capital are often far higher for them than for poorer households".

The concept of 'sin stocks' gives further support to our two central assumptions, namely that social returns matter and that they might have a considerable stochastic component. The literature in this field mainly focusses on whether these morally doubtful stocks are able to outperform in terms of financial returns. Fabozzi, Ma, and Oliphant (2008, p.83) present "empirical evidence that shows sin stocks have outperformed the market on a risk-adjusted basis". First, this result implies that investors do care about social returns. Sin stocks have to offer investors a financial premium to compensate for the 'social harm' done by the investment, vice versa for 'virtue investments'. Second, if a high financial yield of such a company stems from high turnover, their social returns are low when financial returns are high, i.e., there is a negative (intra-asset) covariance, which is an argument in favor of modeling stochastic social returns. This 'success channel' also works the other way round for virtue investing: A solar energy producer has high social returns when output, turnover and profits are high, i.e., a positive intra-asset covariance.

Therefore, we can justify stochastic social returns. However, as mentioned in the introduction, do investors care about variations of social returns? To answer this question, we use the distinction between intrinsic and extrinsic motivation for altruism, as stressed by Sen (1987), for instance. If socially oriented investors are intrinsically motivated, they will care about variations in social returns. After all, they derive utility from the actual results of their investment. Our theoretical model also allows for investors who do care about the expected social return, but not about variations in it (the 'risk-neutral to social returns' investors). This type of investor is extrinsically motivated, investing socially in order to tell colleagues, friends and family about themselves being good guys. In case of corporate investment, it is about telling the public. Their utility stems from telling others, and it arises before (social) returns are actually realized. Therefore, taking the risk of an extreme negative outcome would not decrease their utility much.

Formally, if social returns are stochastic, an investor's objective function depends on two random variables: financial return $R_{P}$ and social return $S_{P}$. In analogy to the financial variables, let $\mu_{S_{i}}$ be the expected social return of asset $i, \sigma_{S_{i}}^{2}$ its social variance and $\sigma_{S_{i}, S_{j}}$ the covariance between social returns of assets $i$ and $j$. If social returns are stochastic, we have cross covariances between financial returns of asset $i$ and social returns of asset $j$, denoted by $\sigma_{R_{i}, S_{j}}$. As with individual assets, we use $\mu_{R_{P}}$ as the expected financial return of the portfolio, $\mu_{S_{P}}$ as its expected social return and $\sigma_{R_{P}}^{2}$ and $\sigma_{S_{P}}^{2}$ as the respective variances of the portfolio. The portfolio covariance is $\sigma_{R_{P}, S_{P}}$.

\subsubsection{Preference axiomatics}

The investor's objective function should be such that it satisfies the following axioms on preferences. Consider an investor facing two portfolios. First, if both portfolios have identical financial and social 
means and variances and the same covariance, the investor should be indifferent between them, i.e., portfolio choice should not depend on anything else than these five moments. Second, if two portfolios only differ in financial mean, a rational investor should prefer the one with the higher mean. Third, the one with the higher social mean should be preferred if the portfolios only differ in social mean. Fourth, risk-averse investors should choose the portfolio with the lower financial variance if all other things are equal and, fifth, the same shall be true for two portfolios differing only in social variance. Sixth, since both financial and social returns are a good thing, it makes sense to assume correlation aversion as in Epstein and Tanny (1980). This means that between two portfolios with the same means and variances, the one with the lower (more negative) covariance shall be preferred. A portfolio with a strong positive covariance is dominated since it pays a low social return exactly when financial returns are low, too. If the covariance is negative, an investor can take comfort in high social returns in times of low financial returns.

Clearly, portfolio choice is an ex ante choice, actual financial and social returns of the portfolio cannot be used to determine the shares of wealth invested in the assets. We can only work with statistical moments. From the literature on the theory of choice under uncertainty, the most common approach is to specify a utility function and then let investors maximize expected utility. As an alternative, mean-variance analysis gets by with less sophisticated empirical estimates and enjoys great popularity in the finance literature. As is well known, there are ways to guarantee consistency between expected utility and mean-variance in a univariate decision framework. In appendix 5.1, we review these conditions for the univariate case and make a proposal on how to align them in case of a bivariate choice problem, as encountered in our model.

In traditional portfolio theory with only financial returns, Markowitz (1952, p. 82) defines the efficient frontier as the subset of all those mean-variance (E-V) combinations "...with minimum $\mathrm{V}$ for given $\mathrm{E}$ or more and maximum $\mathrm{E}$ for given $\mathrm{V}$ or less". Since taking the square root is a monotonically increasing transformation, using the standard deviation instead of the variance in Markowitz' definition yields the same subset of portfolios. Thus, given the above axioms on preferences, we are now able to define the new efficient frontier as follows.

Definition 1: Portfolio A is " $\mu_{R^{-}} \mu_{S^{-}} \sigma_{S^{-}} \sigma_{R^{-}} \sigma_{R, S^{-}}$efficient" if there is no portfolio $B$ with

- $\mu_{R_{A}} \leq \mu_{R_{B}}$,

- $\mu_{S_{A}} \leq \mu_{S_{B}}$,

- $\sigma_{R_{A}} \geq \sigma_{R_{B}}$

- $\sigma_{S_{A}} \geq \sigma_{S_{B}}$,

- $\sigma_{R_{A}, S_{A}} \geq \sigma_{R_{B}, S_{B}}$,

at least one inequality strict.

In words, portfolio A is efficient if there is no other portfolio B with at least as high a financial return, at least as high a social return, at most as high a financial risk, at most as high a social risk, and at most as high an $R-S$ covariance, at least one variable of portfolio B strictly better. In other words, a portfolio is efficient if there is no other, dominant portfolio. 


\subsubsection{The investor's optimization problem}

Given Definition 1, the most intuitive way to translate it into an objective function is to simply sum up the five variables and attach weights to them, i.e., to set up an additive preference function

$$
\beta_{1} \mu_{R_{P}}(\vec{x})+\beta_{2} \mu_{S_{P}}(\vec{x})-\beta_{3} \sigma_{R_{P}}^{2}(\vec{x})-\beta_{4} \sigma_{S_{P}}^{2}(\vec{x})-\beta_{5} \sigma_{R_{P}, S_{P}}(\vec{x}),
$$

with $\beta_{i} \geq 0 .{ }^{8}$ For ease of mathematical treatment the standard deviation terms are squared again. Variations in $\beta_{i}$ represent changes in preferences. In particular, we have

- $\beta_{2}=\beta_{4}=\beta_{5}=0$ : representing a Markowitz investor, social blindness,

- $\beta_{1}=\beta_{3}=\beta_{5}=0$ : representing an altruist, social fanatic,

- $\beta_{1}=\beta_{2}=\beta_{4}=\beta_{5}=0, \beta_{3} \neq 0$ : representing maximum financial risk aversion. ${ }^{9}$

Note that equation (2) is only one possible objective function consistent with Definition 1, and that any objective function consistent with Definition 1 leads to the same efficient frontier. Thus, the efficient frontier can be found through the maximization problem

$$
\begin{gathered}
\max _{\vec{x}}\left\{\beta_{1} \mu_{R_{P}}(\vec{x})+\beta_{2} \mu_{S_{P}}(\vec{x})-\beta_{3} \sigma_{R_{P}}^{2}(\vec{x})-\beta_{4} \sigma_{S_{P}}^{2}(\vec{x})-\beta_{5} \sigma_{R_{P}, S_{P}}(\vec{x})\right\}, \\
\text { s.t. } \sum_{i=1}^{N} x_{i}=1 .
\end{gathered}
$$

The variables in (3) follow from applying standard variance and covariance techniques to the financial and social returns of the portfolio in equation (1):

$$
\begin{gathered}
\mu_{R_{P}}=\sum_{i} x_{i} \mu_{R_{i}} \quad \text { and } \quad \mu_{S_{P}}=\sum_{i} x_{i} \mu_{S_{i}}, \\
\sigma_{R_{P}}^{2}=\vec{x}^{T} \Sigma_{R} \vec{x} \quad \text { and } \quad \sigma_{S_{P}}^{2}=\vec{x}^{T} \Sigma_{S} \vec{x} \\
\sigma_{R_{P}, S_{P}}=\vec{x}^{T} \Sigma_{R S} \vec{x} .
\end{gathered}
$$

The matrices $\Sigma_{R}, \Sigma_{S}$, and $\Sigma_{R S}$ are the covariance matrices

$$
\Sigma_{R}:=\left(\begin{array}{ccc}
\sigma_{R_{1}, R_{1}} & \ldots & \sigma_{R_{1}, R_{N}} \\
\vdots & \ddots & \vdots \\
\sigma_{R_{N}, R_{1}} & \ldots & \sigma_{R_{N}, R_{N}}
\end{array}\right), \quad \Sigma_{S}:=\left(\begin{array}{ccc}
\sigma_{S_{1}, S_{1}} & \ldots & \sigma_{S_{1}, S_{N}} \\
\vdots & \ddots & \vdots \\
\sigma_{S_{N}, S_{1}} & \ldots & \sigma_{S_{N}, S_{N}}
\end{array}\right)
$$

\footnotetext{
${ }^{8}$ The preference function is over-parameterized, but we keep all five parameters for ease of interpretation at this point of the paper.

${ }^{9}$ In this case only a negative multiple of the financial variance is maximized.
} 


$$
\Sigma_{R S}:=\left(\begin{array}{ccc}
\sigma_{R_{1}, S_{1}} & \ldots & \sigma_{R_{1}, S_{N}} \\
\vdots & \ddots & \vdots \\
\sigma_{R_{N}, S_{1}} & \ldots & \sigma_{R_{N}, S_{N}}
\end{array}\right)
$$

The formula for $\sigma_{R_{P}, S_{P}}$ deserves special attention. Apart from the vector $\vec{x}$, it consists of $\Sigma_{R S}$, which is a matrix containing two sorts of covariances: intra-asset covariances and inter-asset cross covariances.

The diagonal elements $\sigma_{R_{i}, S_{i}}$ of the matrix $\Sigma_{R S}$ are the intra-asset covariances, i.e., the dependency between financial and social returns of asset $i$. Having called the covariance between financial (or social) returns of different assets the 'normal inter-asset covariance', the off-diagonal elements of $\Sigma_{R S}$ can be called 'inter-asset cross covariances'. They give the (degree of linear) dependency between social returns of one asset and financial returns of another. In the case of two assets, there are six covariances in total, as shown in Figure 1. Note that the normal inter-asset covariances $\sigma_{S_{1}, S_{2}}$ and $\sigma_{R_{1}, R_{2}}$ enter the formulas for the portfolio's financial variance and social variance, respectively.

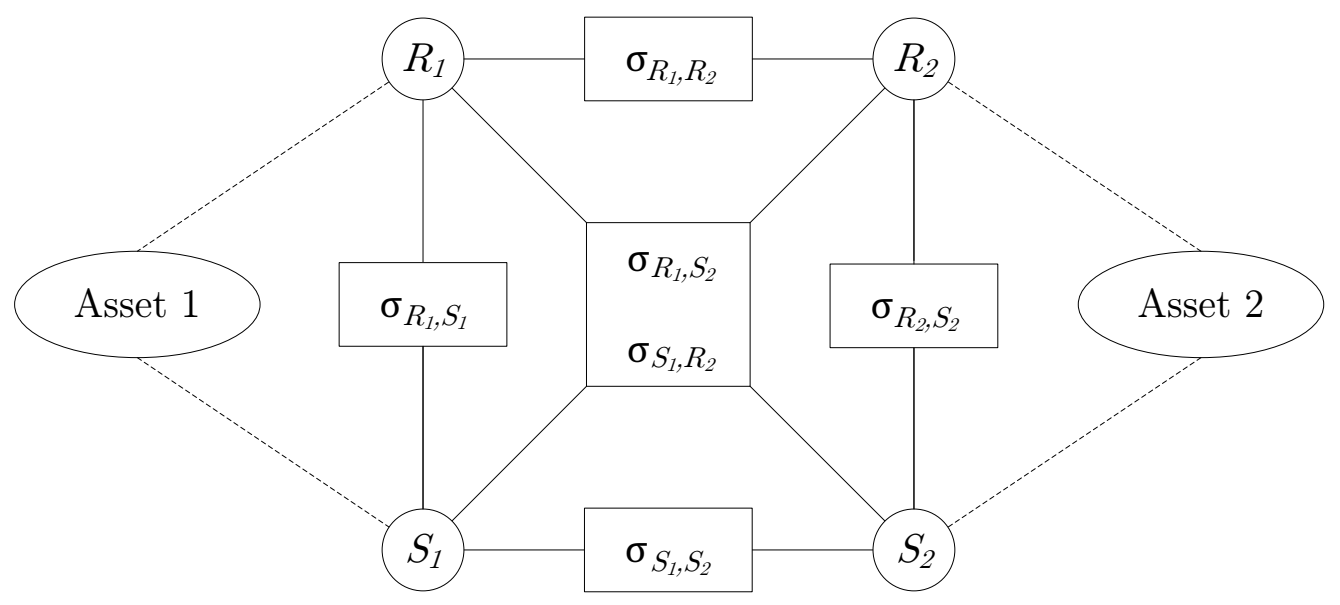

Figure 1: All covariances with 2 assets.

Let us now derive the solution to the optimization problem (3). We define the Lagrangian

$$
L(\vec{x}, \lambda):=\beta_{1} \mu_{R_{P}}(\vec{x})+\beta_{2} \mu_{S_{P}}(\vec{x})-\beta_{3} \sigma_{R_{P}}^{2}(\vec{x})-\beta_{4} \sigma_{S_{P}}^{2}(\vec{x})-\beta_{5} \sigma_{R_{P}, S_{P}}(\vec{x})-\lambda\left(\sum_{i=1}^{N} x_{i}-1\right),
$$

which can be written as

$$
\begin{aligned}
L(\vec{x}, \lambda) & =\beta_{1} \sum_{i=1}^{N} x_{i} \mu_{R_{i}}+\beta_{2} \sum_{i=1}^{N} x_{i} \mu_{S_{i}}-\beta_{3} \sum_{i=1}^{N} \sum_{j=1}^{N} x_{i} x_{j} \sigma_{R_{i}, R_{j}} \\
& -\beta_{4} \sum_{i=1}^{N} \sum_{j=1}^{N} x_{i} x_{j} \sigma_{S_{i}, S_{j}}-\beta_{5} \sum_{i=1}^{N} \sum_{j=1}^{N} x_{i} x_{j} \sigma_{R_{i}, S_{j}}-\lambda\left(\sum_{i=1}^{N} x_{i}-1\right) .
\end{aligned}
$$


The first-order condition (FOC) w.r.t. $x_{i}$ becomes

$$
\frac{\partial L}{\partial x_{i}}=\beta_{1} \mu_{R_{i}}+\beta_{2} \mu_{S_{i}}-2 \beta_{3} \sum_{j=1}^{N} x_{j} \sigma_{R_{i}, R_{j}}-2 \beta_{4} \sum_{j=1}^{N} x_{j} \sigma_{S_{i}, S_{j}}-\beta_{5}\left[\sum_{j=1}^{N} x_{j} \sigma_{R_{i}, S_{j}}+\sum_{j=1}^{N} x_{j} \sigma_{R_{j}, S_{i}}\right]-\lambda,
$$

which must be equal to zero for all assets $i$. The last FOC, $\frac{\partial L}{\partial \lambda}$, is the constraint. Let $\vec{x}^{*}$ contain the optimal asset weights in the portfolio and $\lambda$, so that

$$
\vec{x}^{*}:=\left(x_{1}^{*}, \ldots, x_{2}^{*}, \lambda\right)^{T} .
$$

Moreover, let

$$
\vec{y}:=\left(\beta_{1} \mu_{R_{1}}+\beta_{2} \mu_{S_{1}}, \ldots, \beta_{1} \mu_{R_{N}}+\beta_{2} \mu S_{N}, 1\right)^{T},
$$

and

$$
M:=C_{R}+C_{S}+C_{R S}+C_{R S}^{T} .
$$

The matrices $C_{R}, C_{S}$ and $C_{R S}$ are basically the matrices $\Sigma_{R}, \Sigma_{S}$ and $\Sigma_{R S}$ with an additional row and column each, due to the constraint, and the 'preference coefficients'. ${ }^{10}$

$$
\begin{aligned}
C_{R}:= & \left(\begin{array}{cccc}
2 \beta_{3} \sigma_{R_{1}, R_{1}} & \ldots & 2 \beta_{3} \sigma_{R_{1}, R_{N}} & 1 \\
\vdots & \ddots & \vdots & \vdots \\
2 \beta_{3} \sigma_{R_{N}, R_{1}} & \ldots & 2 \beta_{3} \sigma_{R_{N}, R_{N}} & 1 \\
1 & \ldots & 1 & 0
\end{array}\right), C_{S}:=\left(\begin{array}{cccc}
2 \beta_{4} \sigma_{S_{1}, S_{1}} & \ldots & 2 \beta_{4} \sigma_{S_{1}, S_{N}} & 0 \\
\vdots & \ddots & \vdots & \vdots \\
2 \beta_{4} \sigma_{S_{N}, S_{1}} & \ldots & 2 \beta_{4} \sigma_{S_{N}, S_{N}} & 0 \\
0 & \ldots & 0 & 0
\end{array}\right), \\
C_{R S} & :=\left(\begin{array}{cccc}
\beta_{5} \sigma_{R_{1}, S_{1}} & \ldots & \beta_{5} \sigma_{R_{1}, S_{N}} & 0 \\
\vdots & \ddots & \vdots & \vdots \\
\beta_{5} \sigma_{R_{N}, S_{1}} & \ldots & \beta_{5} \sigma_{R_{N}, S_{N}} & 0 \\
0 & \ldots & 0 & 0
\end{array}\right) .
\end{aligned}
$$

Theorem 1: Solution to the most general case. If $M$ is invertible, then

$$
\vec{x}^{*}=M^{-1} \vec{y}
$$

is the unique solution to (3).

The matrix $M$ is quadratic, symmetric and of dimension $N+1$. Given invertibility of $M$, it is easy to see that (13) is the solution of (3). Multiplying equation (13) with $M$ from the left and transforming the resulting equation to the underlying system of equations gives the $(N+1)$ FOCs.

The efficient frontier under the preference axiomatics is given in parametric form in equation (13). As can be seen from equations (11) and (12), the solution depends on the five preference parameters $\beta_{1}, \ldots, \beta_{5}$. Let $\mathbb{P}=\left\{\beta_{1} \times \ldots \times \beta_{5}\right\}$ be the Cartesian product of preference coefficients. Thus, $\vec{x}^{*}$ is a mapping of $\mathbb{P}$ to $\mathbb{E}$, the latter being the set of efficient portfolios (with $\mathbb{E} \subset \mathbb{R}^{5}$ ). If we were able

$\overline{10}$ We could have added the row and column filled with ' 1 ' in $C_{R}$ to any of the other two matrices. 
to plot five-dimensional graphs, we could vary all five parameters from zero to some large number to get a picture of the efficient frontier. In Section 2.2, we assume deterministic social returns. This allows us to derive graphs of the efficient frontier.

\subsection{Deterministic social returns}

In this section, we consider a simplified version of the general optimization problem (3), namely with deterministic social returns. As indicated in the introduction, the resulting optimization problem is similar to the special case of (3) which results from setting both $\beta_{4}$ and $\beta_{5}$ equal to zero. This special case would also describe an investor who faces stochastic social returns but does not condition his optimal decision on the volatility of social returns. Above, we have called this type of investor 'risk-neutral in social returns'.

\subsubsection{Axiomatics with deterministic social return}

Compared to the definition of efficiency in Subsection 2.1.2 (Definition 1), the search for the efficient frontier becomes easier. Since social returns are deterministic, there are neither social variance nor social financial covariance terms, and we write $S_{P}$ instead of $\mu_{S_{P}}$. The efficiency notion simplifies in the following way.

Definition 2: Portfolio A is " $\mu-S-\sigma$ efficient" if there is no portfolio B with

- $\mu_{A} \leq \mu_{B}$,

- $S_{A} \leq S_{B}$,

- $\sigma_{A} \geq \sigma_{B}$,

at least one inequality strict.

Now, the financial mean and variance of the portfolio are called $\mu_{P}$ and $\sigma_{P}$, respectively. The preference function we use is

$$
\beta_{1} \mu_{P}(\vec{x})+\beta_{2} S_{P}(\vec{x})-\sigma_{P}^{2}(\vec{x}),
$$

normalizing the financial risk coefficient, $\beta_{3}=1$.

As in Subsection 2.1, we can capture different types of investors by varying the two preference coefficients. In this subsection, the term 'risk aversion' is unambiguously related to financial risk aversion. Even if there is no canonical or straightforward measure of risk aversion in this setup, we can define the notion of one individual being more risk averse than another in the following way: A tuple $T=\left(\beta_{1}, \beta_{2}\right)$ represents higher risk aversion than a tuple $T^{\prime}=\left(\beta_{1}^{\prime}, \beta_{2}^{\prime}\right)$ if $\beta_{1} \leq \beta_{1}^{\prime}$ and $\beta_{2} \leq \beta_{2}^{\prime}$, with strict inequality for one of them. Thus, preferences we are able to model include

- $\beta_{1}=0$ : financial mean blindness,

- $\beta_{2}=0$ : social blindness,

- $\beta_{1}=\beta_{2}=0$ : maximum risk aversion,

- $\beta_{1} \rightarrow \infty$ and $/$ or $\beta_{2} \rightarrow \infty$ : risk neutrality. 
If, in addition to the normalized coefficient, one coefficient is fixed, the other determines the preference trade-off between the remaining two variables. ${ }^{11}$ The preference set $\mathbb{P}$ is now a two-dimensional one such that the optimal solution $\vec{x}^{*}$ becomes a mapping from two into three-dimensional space. This enables us to plot the efficient frontier. We do this in the application using real world data. To find the efficient frontier, we consider the simplified optimization problem

$$
\begin{gathered}
\max _{\vec{x}}\left\{\beta_{1} \mu_{P}(\vec{x})+\beta_{2} S_{P}(\vec{x})-\sigma_{P}^{2}(\vec{x})\right\}, \\
\text { s.t. } \sum_{i=1}^{N} x_{i}=1 .
\end{gathered}
$$

The solution is already given by equation (13). The matrix $M$ becomes the matrix $C_{R}$ with $\beta_{3}=1$ since $C_{S}$ and $C_{R S}$ consist of only zeros in the deterministic case (or, if investors do not care about variations in social returns, $\beta_{4}=\beta_{5}=0$ make them consist of only zeros). Let

$$
C_{R}^{-1}:=K:=\left(\begin{array}{ccc}
k_{1,1} & \ldots & k_{1, N+1} \\
\vdots & \ddots & \vdots \\
k_{N+1,1} & \ldots & k_{N+1, N+1}
\end{array}\right)
$$

be the inverse of $C_{R}$. Thus, we can write the components of the solution vector $\vec{x}^{*}$, which maximizes (14), as

$$
x_{i}^{*}=\sum_{j=1}^{N} k_{i, j}\left(\beta_{1} \mu_{R_{j}}+\beta_{2} S_{j}\right)+k_{i, N+1} .
$$

The formula is also valid for $x_{N+1}^{*}=\lambda$.

The vector $\vec{x}^{*}$ is a function of the remaining two preference coefficients $\beta_{1}$ and $\beta_{2}$. Inserting the optimal solution $\vec{x}^{*}$ (without $x_{N+1}^{*}=\lambda$ ) into the three portfolio dimensions financial mean, social return and financial risk, the optimal portfolio is a triple depending on two parameters, $\beta_{1}$ and $\beta_{2}$. The general procedure of optimization also allows for a riskless asset, simply by choosing the characteristics of one of the assets such that it equals the riskless asset, i.e., zero risk and some positive financial return (and a suitable social return). Another way to include a riskless asset is to reformulate the optimization problem, which we do in Appendix 5.2.

\subsubsection{Tobin-like separation}

This section is concerned with Tobin-like separation properties, i.e. the division of wealth into a riskless asset on the one hand and a risky portfolio on the other. Tobin (1958) proved that the composition of the tangent portfolio is independent of $\mu$ - $\sigma$-preferences. In his setup, differences in risk aversion influence only the allocation between the riskless asset and the risky (tangent) portfolio.

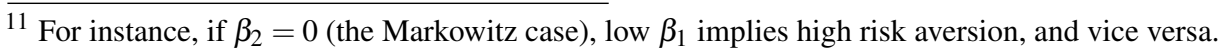


Definition 3: Preferences of two investors, represented by two tuples $T=\left(\beta_{1}, \beta_{2}\right)$ and $T^{\prime}=\left(\beta_{1}^{\prime}, \beta_{2}^{\prime}\right)$ are said to only differ in risk aversion if, and only if, $\frac{\beta_{1}}{\beta_{2}}=\frac{\beta_{1}^{\prime}}{\beta_{2}^{\prime}}$ and $\beta_{j} \neq \beta_{j}^{\prime}$ for $j=1,2$ and $\beta_{2}, \beta_{2}^{\prime}>0$.

The next theorem makes a statement on the possibility of Tobin separation.

Theorem 2: No Tobin separation in the deterministic setup. There is no unique tangent portfolio. The composition of the optimal portfolio of risky assets depends on preferences.

Proof: To include a riskless asset in the analysis, we can simply assign the characteristics of the riskless asset to one of our $i$ assets. Let $x_{1}$ be the share of wealth invested in the riskless asset. This determines some values in the matrices $C_{R}$ and $K=C_{R}^{-1}$. Clearly, since there is no variation in the return of asset 1 , its variance and the covariance with all other assets is zero. Therefore, $\sigma_{R_{1}, R_{j}}=$ $\sigma_{R_{i}, R_{1}}=0$ for all $i, j=1, \ldots, N$. These changes also affect the inverse of $C_{R}: k_{i, N+1}=k_{N+1, j}=0$ for all $i, j \geq 2$ and $k_{1, N+1}=k_{N+1,1}=1$. Also, we take the riskless asset to have a social return of zero so that $S_{1}=0$.

The portfolio of risky assets consists of the assets $i=2, \ldots, N$. The weight of each individual asset in that portfolio (i.e., for $i \geq 2$ ) can be calculated as

$$
x_{i}^{T P}=\frac{\sum_{j=1}^{N} k_{i, j}\left(\beta_{1} \mu_{R_{j}}+\beta_{2} S_{j}\right)+k_{i, N+1}}{\sum_{m=2}^{N} \sum_{j=1}^{N} k_{m, j}\left(\beta_{1} \mu_{R_{j}}+\beta_{2} S_{j}\right)+k_{m, N+1}} .
$$

It is important to note that normalizing $\beta_{3}$ to unity implies that $C_{R}$ and, thus, $K$ do not depend on preference coefficients. We can clearly see that variations in $\beta_{1}$ and $\beta_{2}$ influence the weights of the risky assets in the tangent portfolio.

q.e.d.

Theorem 3: Tobin-like separation in the deterministic setup with a riskless asset. The composition of the tangent portfolio is identical for investors who only differ in risk aversion.

Proof: Since $k_{i, N+1}=0$ for all $i \geq 2$, rearranging equation (16), namely by separating sums and dividing numerator and denominator by $\beta_{2}(>0)$, yields:

$$
x_{i}^{T P}=\frac{\frac{\beta_{1}}{\beta_{2}} \sum_{j=1}^{N} k_{i, j} \mu_{R_{j}}+\sum_{j=1}^{N} k_{i, j} S_{j}}{\frac{\beta_{1}}{\beta_{2}} \sum_{m=2}^{N} \sum_{j=1}^{N} k_{m, j} \mu_{R_{j}}+\sum_{m=2}^{N} \sum_{j=1}^{N} k_{m, j} S_{j}} .
$$

Thus, variations in $\beta_{1}$ and $\beta_{2}$ can clearly change the weights in the tangent portfolio. However, a change only in risk aversion according to Definition 3 means that we scale both $\beta_{1}$ and $\beta_{2}$ up or down by a constant so that the ratio $\frac{\beta_{1}}{\beta_{2}}$ does not change. From equation (17), it is only this ratio which influences the weights in the tangent portfolio, not their absolute values.

q.e.d. 
Theorem 3 does not imply that the absolute amounts of wealth invested in each of the risky assets do not change. Only the relative weights of the risky assets within the optimal portfolio of risky assets do not change. To see the latter point more clearly, consider the share of total wealth invested in the riskless asset.

$$
x_{1}=\sum_{j=1}^{N} k_{1, j}\left(\beta_{1} \mu_{j}+\beta_{2} S_{j}\right)+1=1+\beta_{1} \sum_{j=1}^{N} k_{1, j} \mu_{j}+\beta_{2} \sum_{j=1}^{N} k_{1, j} S_{j}
$$

since $k_{1, N+1}=1$. If we scale both $\beta_{1}$ and $\beta_{2}$ up or down such that their ratio $\frac{\beta_{1}}{\beta_{2}}$ does not change, $x_{1}$ does change.

In the remainder of this section, we try to give an intuition for our separation properties at the expense of formal rigor. In a Markowitz world, investors care about financial returns only. Thus, their trade-off between return and risk is described by the Sharpe ratio expressing how much expected excess return one can get per unit of risk borne. Investors try to maximize this financial risk premium. Since both excess return and risk (using the standard deviation) are linear functions of the share of wealth invested in the riskless asset, shifting funds between the riskless asset and any given portfolio does not change the Sharpe ratio. Thus, it is the same portfolio of risky assets which all investors choose - the one that maximizes the Sharpe ratio.

Once we include social returns, the trade-off between return and risk becomes more difficult. For bearing risk investors can be compensated by either a financial or a social premium, or a mixture between the two. Let the 'social financial Sharpe ratio' (SFSR) be given by

$$
S F S R=\frac{\beta_{1}\left(\mu_{P_{\text {all }}}-r\right)+\beta_{2}\left(S_{P_{a l l}}-0\right)}{\sigma_{P_{\text {all }}}},
$$

where $\mu_{P_{\text {all }}}, S_{P_{\text {all }}}$ and $\sigma_{P_{\text {all }}}$ are the financial mean, social return and standard deviation of a portfolio built from risky assets and a riskless asset, respectively. From a look at the objective function in (14), it is clear that increases in risk must be traded-off against increases in the terms which enter the objective function positively. The premium for risk is then $\beta_{1} \mu_{P_{\text {all }}}+\beta_{2} S_{P_{\text {all }}}-\beta_{1} r-\beta_{2} \cdot 0$, or, after rearranging, the numerator of $S F S R$. Since $S_{P_{a l l}}$ is also linear in the share of wealth invested in the riskless asset, reallocations of funds between the riskless asset and any portfolio do not change the SFSR for given $\beta_{1}$ and $\beta_{2}$. So if we compare two investors which differ in risk aversion only, the less risk-averse investors try to maximize an SFSR which is a multiple of the more risk-averse investors' SFSR. Thus, their optimal portfolios are identical. However, this is clearly not true for two investors which also differ in their relative appraisal for social and financial returns.

To summarize, even in the deterministic setup, there is no unique tangent portfolio, but a set of tangent portfolios instead. However, we could speak of a conditional tangent portfolio, conditionality with respect to the relative financial mean and social return preferences: Fixing the ratio $\frac{\beta_{1}}{\beta_{2}}$ leads to a single portfolio of risky assets, irrespective of changes in risk aversion. Given such a ratio, asset allocation is a binary problem: How much is to be invested in the riskless asset and how much in the tangent portfolio. 


\section{Application}

\subsection{Deterministic social returns: a socially-minded private investor}

In Subsection 2.2 we consider a portfolio optimization problem with deterministic social returns. In this section we present an application of the deterministic setup, looking at an investor who faces a limited investment opportunity set, consisting of three asset classes: equities (S\&P Euro), bonds (EuroMTS Global Index), and a microfinance investment fund (MFIF, here: responsAbility Global Microfinance Fund).

\subsubsection{Data}

Data on the S\&P Euro Index, the EuroMTS Global Index and the responsAbility Global Microfinance Fund are obtained from Thomson Reuters Datastream database. Since we do not have enough annual observations of the MFIF (03/2005 to 10/2009), we use monthly prices to derive financial returns. We use the maximum of data available for each index (until 10/2009) to derive estimates on expected returns, variances and covariances, and then annualize. In terms of social returns, we assign zero return to all but the MFIF. Therefore, a change in absolute value of social returns for the MFIF does not change the optimal allocation (given that the preference coefficient for social returns changes accordingly). We choose 0.01. Descriptive statistics are given in Table 1.

\begin{tabular}{lrrr}
\hline & equity index & bond index & MFIF \\
\hline$\mu_{R_{i}}$ & 0.1101 & 0.0458 & 0.0405 \\
$S_{i}$ & 0.0000 & 0.0000 & 0.0100 \\
$\sigma_{R_{i}}$ & 0.1853 & 0.0344 & 0.0129 \\
\hline Correlations & & & \\
\hline equity index & 1.0000 & & \\
bond index & -0.2117 & 1.0000 & \\
MFIF & -0.3217 & 0.1027 & 1.0000 \\
\hline
\end{tabular}

Table 1: Descriptive statistics: expected financial returns, social returns, standard deviations, and correlations.

\subsubsection{Results}

Results are given graphically. Figure 2 shows the set of efficient portfolios in three-dimensional space. All four pictures in it describe the same graph. Optimization is done without a riskless asset. The three small (two-dimensional) pictures in Figure 2 are rotated such that one of the three dimensions disappears.

The first of the small graphs is the well-known picture with mean and standard deviation on the ordinate and abscissa, respectively. It is similar in shape to efficient frontiers based only on financial characteristics. However, it is a surface and not a curve. In a purely financial world, only the upper 

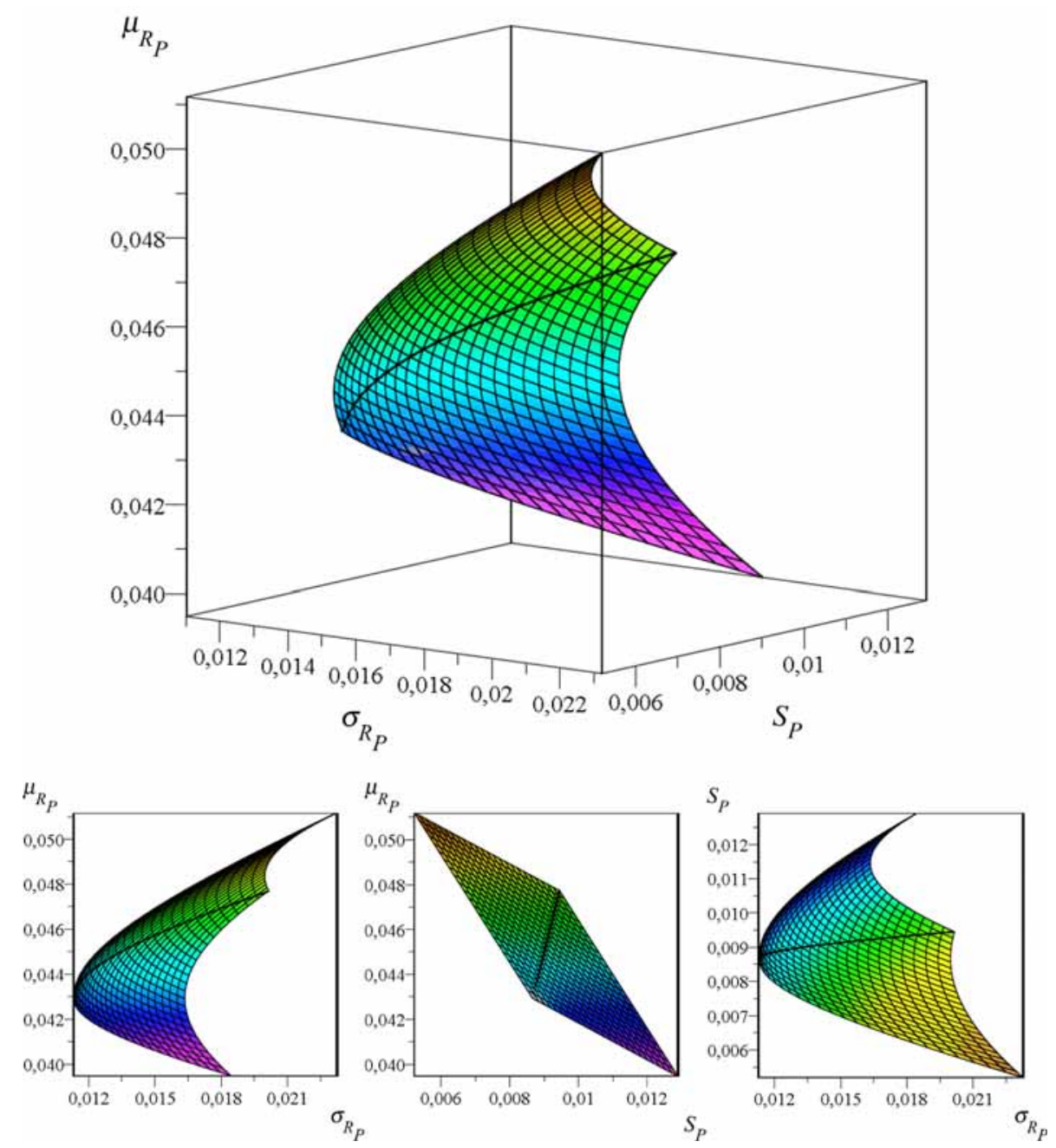

Figure 2: Three-dimensional efficient frontier for the example with deterministic social returns. The black line represents $\beta_{1}=\beta_{2}$.

left frontier of that surface would be efficient. Since we have another dimension, viz., social returns, the "inefficient mean-variance portfolios" (the off-frontier portfolios in the small graph on the left) are " $\mu-S-\sigma$ efficient". Even though they are dominated in terms of expected financial return and variance, high social returns make them optimal for a rather socially oriented investor. The same kind of thoughts explains the surfaces in the remaining two small graphs in Figure 2. The line in Figure 2 represents the set of efficient portfolios for $\beta_{1}=\beta_{2}$. Having normalized $\beta_{3}=1$, this means that there is a change in preferences: Increasing betas represent a less risk-averse investor, and vice versa. ${ }^{12}$ In particular, comparing risk aversion to financial return preferences, the investor puts more emphasis on expected financial return, and, comparing risk aversion and social returns, the investor puts more emphasis on social returns.

\footnotetext{
12 Note that this does not contradict Theorem 3. Since optimization underlying this figure is done without a riskless asset, changes solely in risk aversion now do change the composition of the optimal portfolio.
} 
Using the same data set, we consider the change in investment behavior from introducing a riskless asset with riskless financial return of 0.04. Figure 3 shows the new efficient frontier, best described as a cone section with the vertex being where the riskless asset is located.
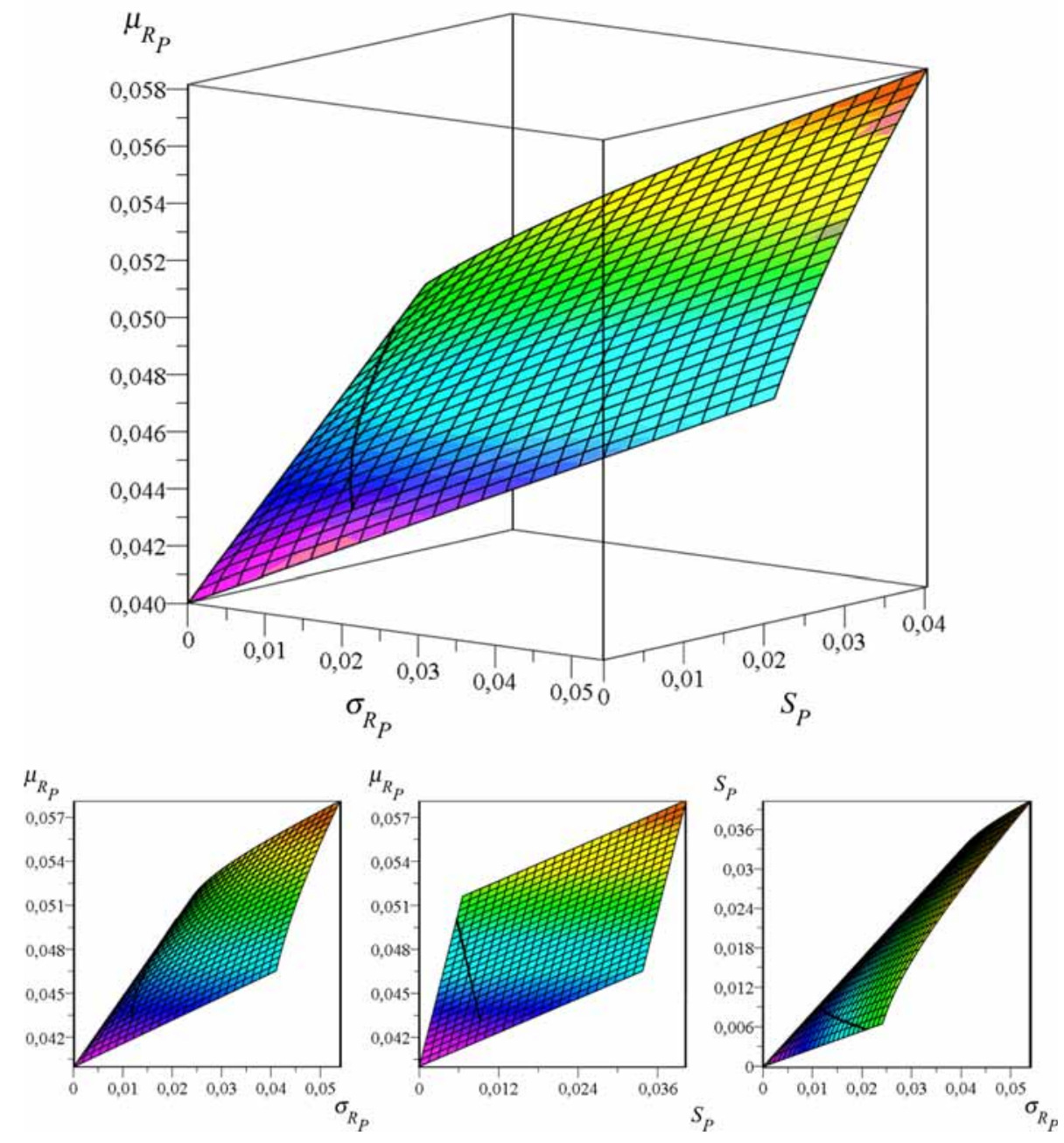

Figure 3: Set of efficient portfolios with riskless asset for the example with deterministic social returns. The black line represents the tangent portfolios for $\beta_{1}=0.005$ and $\beta_{2} \in[0 ; 0.1]$.

Tobin-like separation can also be seen in Figure 3. To get that graph, we fix $\beta_{1}$ to 0.005 and vary $\beta_{2}$ from 0 to 0.01 . The resulting curve in three-dimensional space is the locus of tangential portfolios. This is the visualization of Theorem 2: There is no tangent portfolio. Had we varied both $\beta_{1}$ and $\beta_{2}$ such that they are always equal in absolute value, the result would be a point in three-dimensional space. This would be one way to visualize Theorem 3: The composition of the tangent portfolio is identical for investors who only differ in risk aversion. Another way to visualize the theorem is to plot the weights of the individual assets in the efficient portfolio in two-dimensional space. 


\subsection{Stochastic social returns: microfinance}

In this section we demonstrate an application of the general optimization problem discussed in Subsection 2.1. We look at an MFIF which wants to make equity investments and has to choose optimal weights of the MFIs to be funded. We restrict our investigation to five MFIs: Alexandria Business Association (ABA, Egypt), BURO (Bangladesh), D-miro (Ecuador), FINCA (Peru), and Negros Women for Tomorrow Foundation (NWTF, Philippines). The selection criteria for the MFIs are explained in section 3.2.1.

\subsubsection{Data}

Data on the MFIs are collected from MIX Market database. MIX Market is a web-based information platform by the Microfinance Information Exchange, an organization which provides annual data on financial and social performance of MFIs, MFIFs and microfinance networks worldwide. MIX Market uses a diamond ranking system to indicate the level of disclosure of the MFIs. Five is the maximum number of diamonds an MFI can get. As a proxy for the financial return we use the Return on Equity (ROE). Among the MFIs with five diamonds we consider only MFIs with enough data to compute the ROE for ten successive years (1999-2008). For the calculation of the ROE of the 26 remaining MFIs we convert into euro and use the same formula as MIX Market: net operating income, less taxes divided by average equity. We choose five MFIs with rather moderate ROEs.

We construct a ratio of two variables as a basic proxy for social returns of an MFI: number of active borrowers divided by average assets. The basic idea is that an MFI with many small loans serves poorer clients, having a stronger impact. Thus, average loan size (total loan portfolio outstanding divided by number of active borrowers) is frequently taken as a proxy for social performance. However, since we work with return on equity (ROE) on the financial side, it seems more consistent to consider the respective social return on a per capital unit base, too. According to Rhyne (2009, p. 162), the number of active clients is a commonly used proxy for social performance.

We then estimate expected financial and social returns, financial and social variances, covariances of financial returns and covariances of social returns, as well as the inter-asset cross covariances. Table 2 shows the results.

\subsubsection{Results}

In this subsection, for better interpretability we use the preference function

$$
\alpha\left[\mu_{R_{P}}(\vec{x})+\gamma \mu_{S_{P}}(\vec{x})\right]-\sigma_{R_{P}}^{2}(\vec{x})-\gamma^{2} \sigma_{S_{P}}^{2}(\vec{x})-\gamma \sigma_{R_{P}, S_{P}}(\vec{x}) .
$$

Preference function (20) is the result of a parsimonious model of the investor's preferences in the following sense. First, the investor builds an aggregated total return $R_{P}+\gamma S_{P}$ comprising the financial and the social return where $\gamma$ measures the importance of the social returns compared to the financial returns. In a second step the usual $\mu-\sigma^{2}$ calculus is applied to the aggregated return with $\frac{1}{\alpha}$ measuring the risk aversion with respect to the aggregated return's variance. With this approach 


\begin{tabular}{lrrrrr}
\hline & ABA & BURO & D-miro & FINCA & NWTF \\
\hline$\mu_{R_{i}}$ & 0.0542 & 0.1171 & 0.0864 & 0.0669 & 0.0421 \\
$\sigma_{R_{i}}$ & 0.0257 & 0.0972 & 0.1014 & 0.0481 & 0.0450 \\
$\mu_{S_{i}}$ & 0.0155 & 0.1381 & 0.0252 & 0.0542 & 0.0877 \\
$\sigma_{S_{i}}$ & 0.0070 & 0.0264 & 0.0090 & 0.0050 & 0.0076 \\
\hline Correlations of financial returns & & & & & \\
\hline ABA & 1.0000 & & & & \\
BURO & -0.7973 & 1.0000 & & & \\
D-miro & -0.6786 & 0.8789 & 1.0000 & & \\
FINCA & 0.2289 & -0.2315 & -0.4958 & 1.0000 & \\
NWTF & 0.2770 & 0.0711 & 0.0800 & 0.5402 & 1.0000 \\
\hline Correlations of social returns & & & & & \\
\hline ABA & & & & & \\
BURO & 1.0000 & & & & \\
D-miro & 0.9229 & 1.0000 & & & \\
FINCA & 0.0944 & 0.2239 & 1.0000 & & \\
NWTF & 0.5457 & 0.7625 & 0.0462 & 1.0000 & \\
\hline Inter-asset cross correlations $\left(\rho_{R_{i}, S_{j}}=\frac{\sigma_{R_{i}, S_{j}}}{\sigma_{R_{i}} \sigma_{S_{j}}}\right)$ & -0.4894 & -0.4057 & -0.2942 & 0.0683 & 1.0000 \\
\hline ABA $\left(\rho_{R_{A B A}, S_{j}}\right)$ & & & & & \\
BURO $\left(\rho_{R_{B U R O}, S_{j}}\right)$ & 0.0273 & -0.2304 & -0.3393 & -0.5097 & -0.3109 \\
D-miro $\left(\rho_{R_{\text {Dmiro }}, S_{j}}\right)$ & 0.4507 & 0.6172 & 0.3291 & 0.5678 & -0.1020 \\
FINCA $\left(\rho_{R_{F I N C A}, S_{j}}\right)$ & 0.5225 & 0.7293 & 0.4800 & 0.7065 & -0.1962 \\
NWTF $\left(\rho_{R_{N W T F}, S_{j}}\right)$ & 0.1201 & -0.1235 & -0.2754 & -0.3943 & -0.0563 \\
\hline & 0.7210 & 0.5192 & -0.0002 & 0.0627 & -0.2021 \\
\hline
\end{tabular}

Table 2: Descriptive statistics: expected returns, standard deviations, and correlations.

we are able to express the investor's preferences in a catchy way. It should, however, be noted that proceeding like that has strong implications on the aversion against the three risk dimensions.

Again, the results are given graphically. The Figures 4 and 5 display the optimal portfolio weights in the case with no riskless asset for varying $\alpha$ and varying $\gamma$, respectively. For $\alpha \in[0.0064 ; 0.01]$ and $\gamma \in[1.32 ; 11.8]$, respectively, all weights are positive which is a more realistic situation for MFIs as short-selling is not possible. The results are interesting since one now can see that in Figure 4 for very low $\alpha$-values the weight of BURO is lowest because it has the highest financial and social risk whereas the weight of ABA, the MFI with the lowest financial risk and low social risk, is highest. For decreasing risk aversion the weights of BURO and NWTF, the MFIs with the highest social returns, increase since the importance of social returns is high compared to financial returns for $\gamma=5$. Although BURO has the highest social return it is not the MFI with the highest weight as the high $\gamma$ also leads to high aversion of social risk and correlation. An investor with a lower $\gamma$ and a higher $\alpha$ would prefer a higher weight of BURO. In the left graph in Figure 6 the BURO has the highest weight for $\gamma=1$ and $\alpha \in[0 ; 0.15]$. 

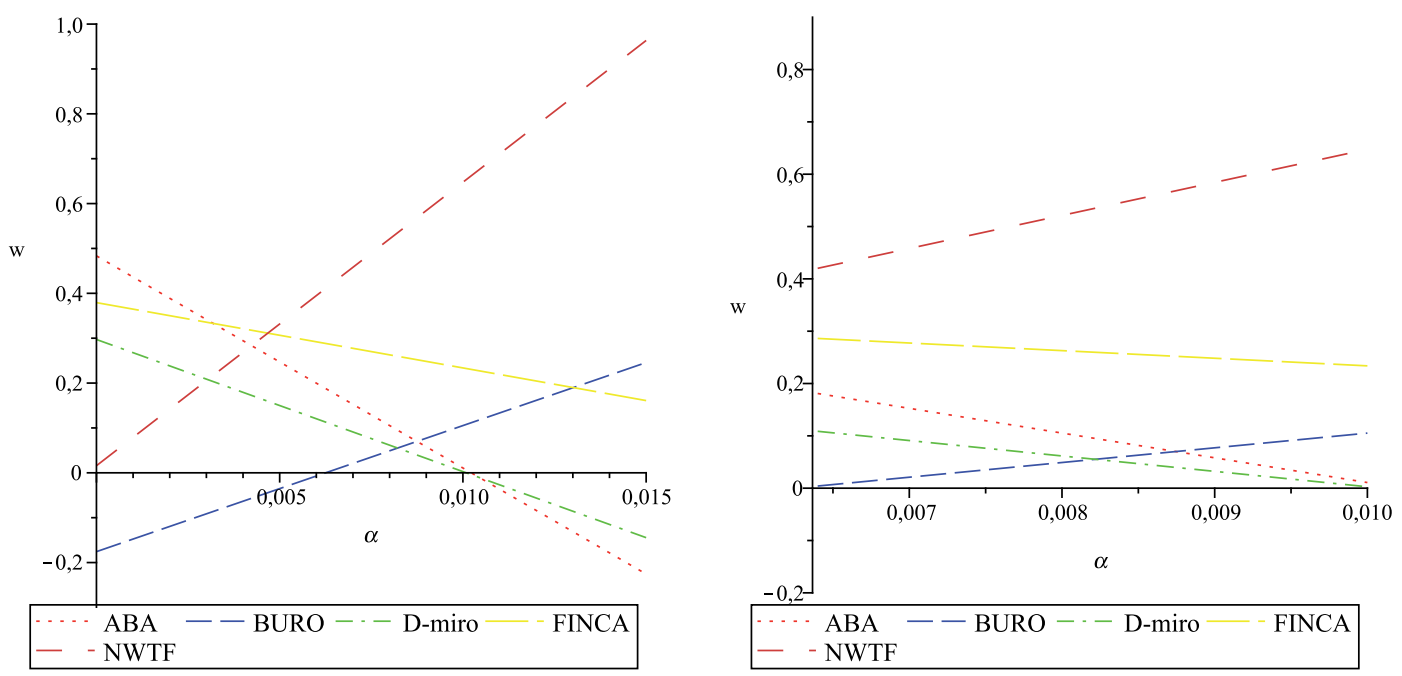

Figure 4: The curves show the optimal weights of all MFIs for the example with stochastic social returns for $\gamma=5$. The left graph shows weights for $\alpha \in[0 ; 0.015]$ and the right graph illustrates weights for $\alpha \in[0.0064 ; 0.01]$. For $\gamma=5$ and $\alpha \in[0.0064 ; 0.01]$ none of the MFIs is shorted because short-selling are not realistic for MFIs.
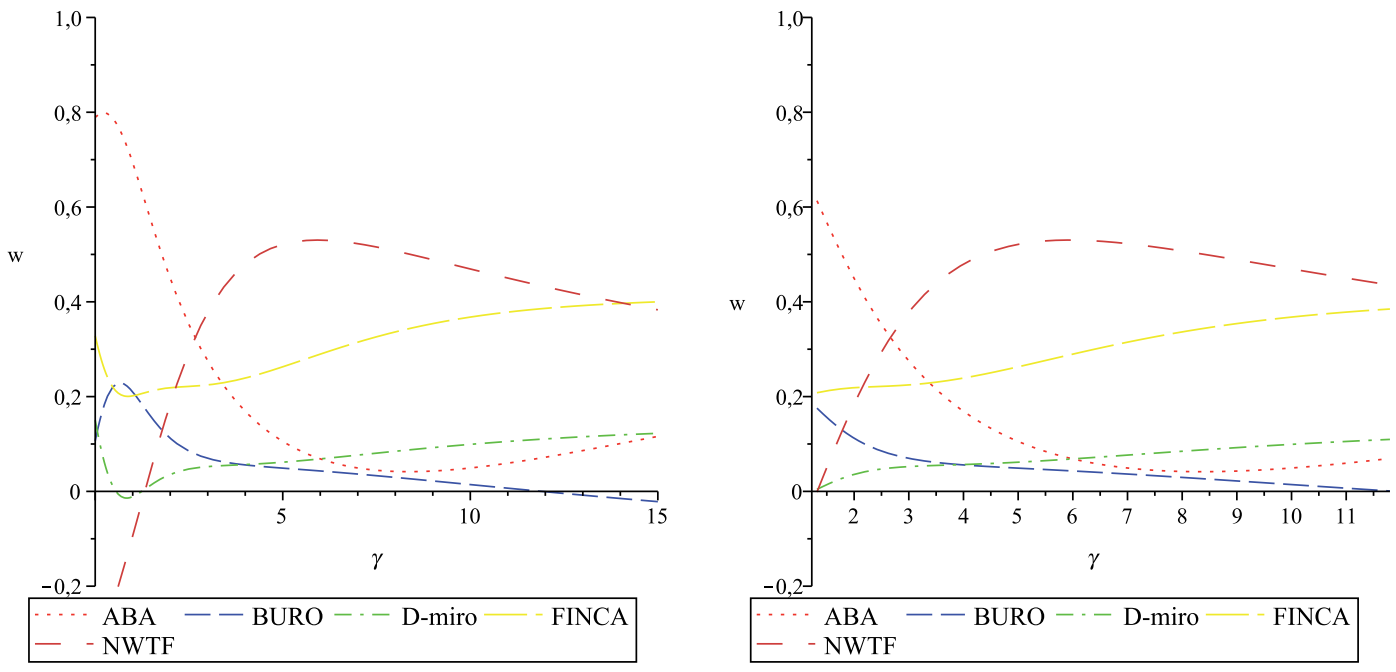

Figure 5: All weights for the example with stochastic social returns for $\alpha=0.008$. The left plot displays weights for $\gamma \in[0 ; 15]$ and the right plot shows weights for $\gamma \in[1.32 ; 11.8]$.

Since we choose $\alpha=0.008$ in Figure 5 in order to get a fairly broad range of solely positive weights of MFIs we deal with a rather risk averse investor. For low $\gamma$-values the weight of ABA is highest because of its low financial and social risk. With increasing $\gamma$ the weight of ABA decreases, since 
it has the lowest social return. However, the weight of ABA increases again until it is highest for very high values of $\gamma$ because, as already mentioned before, a high $\gamma$ also leads to high aversion of social risk and correlation. The weight of FINCA first decreases but then also increases quickly with increasing $\gamma$ as it has a medium social return and the lowest social risk. For very low importance of social returns the weight of NWTF is lowest because ABA has a higher financial return and a lower financial risk. With increasing values of $\gamma$ the weight of NWTF rises fast due to its high social return. However, for very high values of $\gamma$ NWTF has the second lowest weight in the portfolio. The curve of D-miro's weight is similar to FINCA but its weight is lower. Although D-miro has a higher financial return its social return is lower and both financial and social risk is higher. In the case of increasing importance of the social return the weight of BURO first increases and then, presumably due to its high risk in both return dimensions, slowly decreases again. Despite its high social return for $\gamma$ higher than approximately 4 the weight of BURO is lowest because of its high social risk.
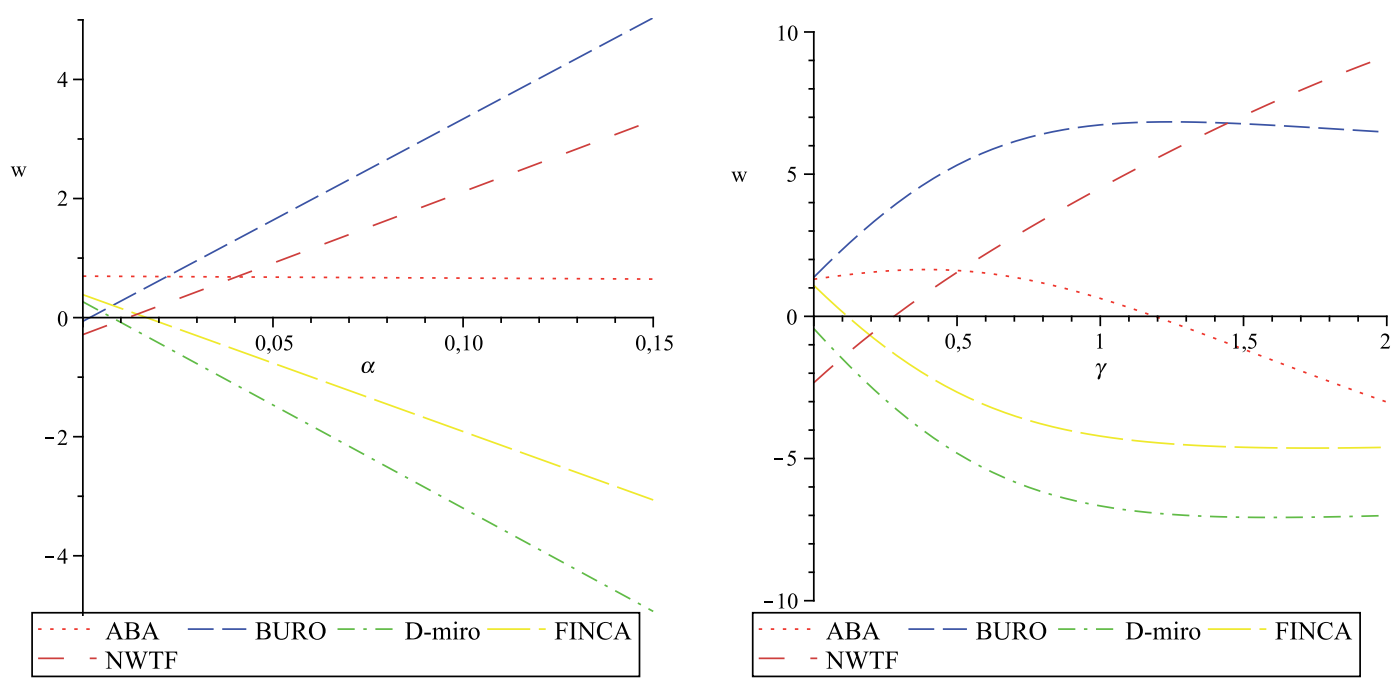

Figure 6: The left plot shows all weights for the example with stochastic social returns for $\gamma=1$ and $\alpha \in[0 ; 0.15]$. The left plot displays weights for $\alpha=0.2$ and $\gamma \in[0 ; 2]$.

Figure 6 shows the portfolio weights of an investor who is less risk averse and has a lower interest in social return. The right plot illustrates that for an MFIF with $\alpha=0.2$ BURO is the MFI with the highest weight for $\gamma \in[0 ; 1.44]$.

The Figures 7 and 8 display the optimal portfolio weights in the case with riskless asset ${ }^{13}$ for varying $\alpha$ and varying $\gamma$, respectively, and show similar results. In Figure 7 one even can see a separation phenomenon which is of course valid in this setting for constant $\gamma$ and varying $\alpha$. With decreasing risk aversion the riskless asset's weight also decreases but slowly increases again as it is riskfree. No separation results hold for varying $\gamma$. However, here also the riskless asset is shorted for $\gamma$ between

13 The riskless asset has a financial return of 0.04 and social return of zero. 


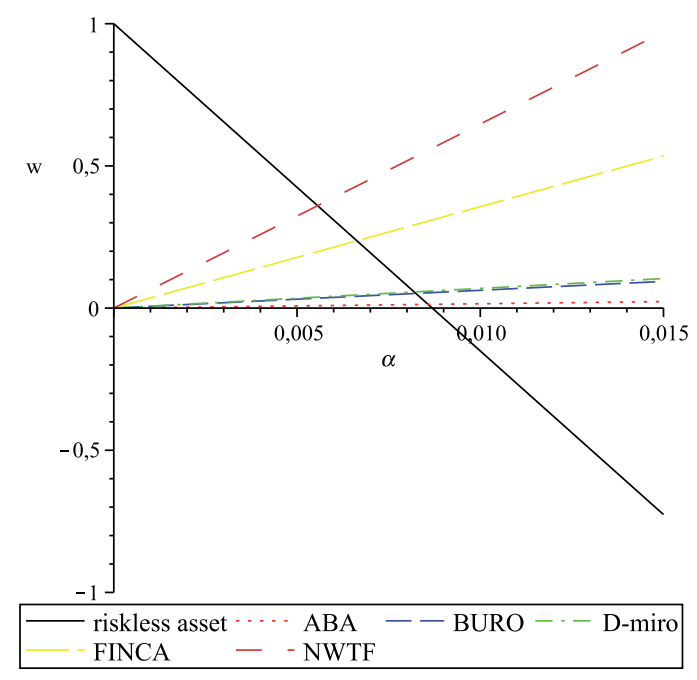

Figure 7: All weights for the example with stochastic social returns with riskless asset for $\gamma=7$ and $\alpha \in[0 ; 0.015]$.
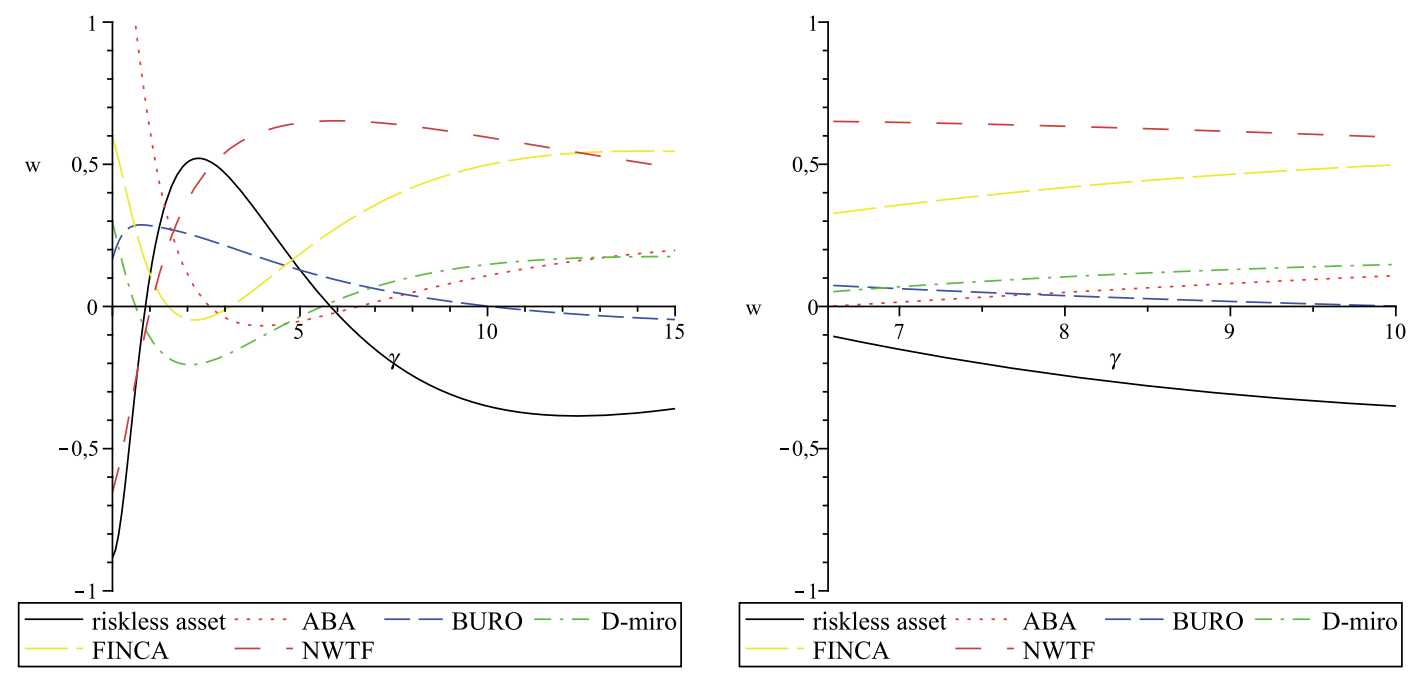

Figure 8: All weights for the example with stochastic social returns with riskless asset for $\alpha=0.01$. The left graph illustrates weights for $\gamma \in[0 ; 15]$ and the right graph shows weights for $\gamma \in[6.6 ; 10]$.

approximately 6 and 28 in order to gain more financial and social return. The weights of the different MFIs are variable. 


\section{Research Outlook and Conclusion}

All we have done so far is just the very first step of the analysis. Several interesting extensions are worth further investigations. In terms of economic theory, a desirable robustness check would consist of incorporating measures of risk other than variance. Also, one should impose short-selling restrictions, i.e., add the constraint $x_{i} \geq 0$ for some $i$ since the theory is applied to investments some of which cannot easily be shorted in reality. This requires the development of an algorithm as in Markowitz (1987).

Furthermore, we think that the inclusion of initial wealth of the investor might yield interesting results. In standard portfolio theory, differences in wealth can result in changes in the preference coefficients $\left(\beta_{i}\right)$. Simply using absolute wealth levels instead of returns in the objective function leads to strange and unrealistic results. In particular, an increase in wealth would increase risk aversion in a mean-variance setup. The consideration of wealth effects becomes more important once social returns are taken into account. Our guess is that higher wealth implies less (financial) risk-aversion and more social concern. A case in point are the high net worth individuals headed by Bill Gates who started to do financially sustainable investment in the past, but mostly change their behavior going into charity.

In terms of application of our model, it is most straightforward to consider further industry funds. However, the measurement of social returns is still very imperfect and difficult. In some industries, people have proposed scales to measure them. In a very small subset of these, there are also data available. The biggest challenge remains the comparison of social returns inter industries. Our model in its most general form with stochastic returns is based on a metric scaling of social returns. But comparing social returns of an arms manufacturer and a microfinance institution is very difficult: How bad is it to produce a hundred of guns and how good is it to provide a hundred of poor children with basic education? And where do we put all the intermediate companies? Where do we put a software company? How close is the development of an e-learning platform to a microfinance investment? And how far away is the software company programming and distributing ego shooters? These are highly normative questions which cannot be answered with a positive analysis of portfolio choice.

And even if it is possible to measure and compare social returns, it will still be hard to incorporate them into the objective function. One way to derive estimates for the values of $\beta_{k}$ is to ask people sophisticated questions which make them reveal their preferences for social returns. Furthermore, it would certainly be interesting to use our model in a macro perspective, e.g., to explain asset prices, or to transfer modern portfolio techniques like the well-known Black-Litterman model (see Black and Litterman (1992)) to the case with social returns. 


\section{Appendix}

\subsection{Expected utility vs. mean-variance}

An excellent survey about mean-variance justifications can be found in Chavas $(2004$, ch.4,6). For univariate optimization, there are three ways to guarantee consistency of mean-variance and expected utility optimization.

At first we consider utility functions defined for levels of final wealth $x$. We will refer to the case of returns as the variable of interest below and start the discussion with quadratic utility

$$
u(x)=a+b x+c x^{2}
$$

with $b>0$ and $c<0$ (risk aversion). Taking expectations yields expected utility

$$
E U(x)=a+b E[x]+c E\left[x^{2}\right]=a+b E[x]+c\left(E[x]^{2}+\operatorname{Var}[x]\right),
$$

which is a function of mean and variance of $x$ only. Clearly, quadratic utility has its drawbacks. For consistency with positive marginal utility, $x$ has to be smaller than $\frac{-b}{2 c}$. Also, risk aversion requires $c<0$ which implies increasing absolute risk aversion (IARA), a highly implausible assumption.

On the other hand, quadratic utility is a useful proxy for many other utility functions since a secondorder Taylor approximation of any differentiable utility function yields a quadratic function.

The second way assumes a specific distribution of wealth, namely a multivariate normal one. In this case, the means, variances and covariances completely describe the probability distribution.

The third way also only assumes a normal distribution of the (univariate) portfolio wealth. In addition, a specific utility is assumed, namely exponential utility

$$
u(x)=-e^{-\gamma x}, \gamma>0 .
$$

This function displays the property of constant absolute risk aversion (CARA), which is more realistic than IARA (as with quadratic utility).

Let $\mu=E[X]$ and $\sigma^{2}=\operatorname{Var}[X]$ be the first two moments of the normally distributed portfolio return $x$. Since $-\gamma x$ is also normally distributed with $E[-\gamma X]=-\gamma \mu$ and $\operatorname{Var}[-\gamma X]=\gamma^{2} \sigma^{2}$ the term $e^{-\gamma X}$ is log-normally distributed. Therefore, the expected utility of $X$ is

$$
E\left[-e^{-\gamma X}\right]=-e^{-\frac{1}{2}\left(-\gamma^{2} \sigma^{2}+2 \gamma \mu\right)}=-e^{-\gamma\left(\mu-\frac{\gamma}{2} \sigma^{2}\right)} .
$$

The last term in the above equation is an increasing function of $\mu-\frac{\gamma}{2} \sigma^{2}$. Therefore, maximization of $\mu-\frac{\gamma}{2} \sigma^{2}$ is equivalent to maximizing expected utility. The latter case is also expandable into the social wealth dimension when using a biattributive utility function of the form

$$
u\left(x_{1}, x_{2}\right)=-e^{-\gamma_{1} x_{1}} \cdot e^{-\gamma_{2} x_{2}}=-e^{-\gamma_{1} x_{1}-\gamma_{2} x_{2}} .
$$

This utility function is of the class $u \circ l$ with $u$ representing a univariate utility function and $l$ a linear form. This class has the comfortable property that the two most important biattributive risk 
aversion concepts coincide (see Dorfleitner and Krapp (2007)). Assuming a normal distribution of $x_{1}$ and $x_{2},-\gamma_{1} x_{1}-\gamma_{2} x_{2}$ is also normally distributed with expectation $-\gamma_{1} \mu_{1}-\gamma_{2} \mu_{2}$ and variance $\gamma_{1}^{2} \sigma_{1}^{2}+\gamma_{2}^{2} \sigma_{2}^{2}+2 \gamma_{1} \gamma_{2} \sigma_{1,2} \cdot{ }^{14}$ Thus, expected utility can be written as

$$
E u\left(x_{1}, x_{2}\right)=-e^{-\gamma_{1} \mu_{1}-\gamma_{2} \mu_{2}+\frac{1}{2} \gamma_{1}^{2} \sigma_{1}^{2}+\frac{1}{2} \gamma_{2}^{2} \sigma_{2}^{2}+\gamma_{1} \gamma_{2} \sigma_{1,2} .}
$$

Since $-e^{-\gamma x}$ is an increasing transformation of $x$, maximization of $\gamma_{1} \mu_{1}+\gamma_{2} \mu_{2}-\frac{1}{2} \gamma_{1}^{2} \sigma_{1}^{2}-\frac{1}{2} \gamma_{2}^{2} \sigma_{2}^{2}-$ $\gamma_{1} \gamma_{2} \sigma_{1,2}$ is identical to maximization of expected utility.

\subsection{Tobin-like separation: an alternative approach}

We again make the simplifying assumption of deterministic social returns. Instead of interpreting one of the assets in our above optimization problem as the riskless one, explicit modeling is even easier. So let $x_{0}$ be the share of wealth invested in the riskless asset, with $x_{0}=1-\sum_{i=1}^{N} x_{i}$ being the residual share. As in the above section, apart from having a variance of zero per se, we assume that the riskless asset has no social return, i.e., $S_{0}=0$.

The portfolio financial mean $\mu_{P}$ becomes

$$
\mu_{P}=r+\sum_{i=1}^{N} x_{i}\left(\mu_{i}-r\right)
$$

The optimization problem is

$$
\max _{x_{1}, \ldots, x_{N}} \beta_{1} r+\beta_{1} \sum_{i=1}^{N} x_{i}\left(\mu_{i}-r\right)+\beta_{2} \sum_{i=1}^{N} x_{i} S_{i}-\sum_{i=1}^{N} \sum_{j=1}^{N} x_{i} x_{j} \sigma_{R_{i}, R_{j}} .
$$

We do not need a constraint about the sum of the asset weights in the portfolio. By construction, optimal weights $x_{0}, \ldots, x_{N}$ add up to one. Again differentiating w.r.t. the weights $\vec{x}$, optimal weights are

$$
\vec{x}^{*}=C^{-1} \times \vec{z},
$$

where $\vec{x}^{*}$ is now of dimension $N$ since there is no constraint. We use

$$
\vec{z}:=\left(\beta_{1}\left(\mu_{1}-r\right)+\beta_{2} S_{1}, \ldots, \beta_{1}\left(\mu_{N}-r\right)+\beta_{2} S_{N}\right)^{T}
$$

(of dimension $N$, too) and the $(N \times N)$ matrix

$$
C:=\left(\begin{array}{ccc}
2 C_{11} & \ldots & 2 C_{1 N} \\
\vdots & \ddots & \vdots \\
2 C_{N 1} & \ldots & 2 C_{N N}
\end{array}\right)=2 \Sigma_{R} .
$$

Let

$$
C^{-1}=K:=\left(\begin{array}{ccc}
k_{11} & \ldots & k_{1 N} \\
\vdots & \ddots & \vdots \\
k_{N 1} & \ldots & k_{N N}
\end{array}\right),
$$

\footnotetext{
$\overline{{ }^{14} \mu_{j} \text { and } \sigma_{j}^{2} \text { are the expectation and variance of }} x_{j}$, respectively. $\sigma_{1,2}$ is the covariance between $x_{1}$ and $x_{2}$.
} 
so that the weights $x_{i}$ for $i=1, \ldots, N$ follow

$$
x_{i}=\beta_{1} \sum_{j=1}^{N} k_{i j}\left(\mu_{j}-r\right)+\beta_{2} \sum_{j=1}^{N} k_{i j} S_{j} .
$$

Excluding the case of $\beta_{2}=0$ (the Markowitz case), we get

$$
x_{i}^{T P}=\frac{\frac{\beta_{1}}{\beta_{2}} \sum_{j=1}^{N} k_{i j}\left(\mu_{j}-r\right)+\sum_{j=1}^{N} k_{i j} S_{j}}{\frac{\beta_{1}}{\beta_{2}} \sum_{m, j=1}^{N} k_{m j}\left(\mu_{j}-r\right)+\sum_{m, j=1}^{N} k_{m j} S_{j}} .
$$

The share of asset $i$ in the optimal portfolio of risky assets only depends on the ratio $\frac{\beta_{1}}{\beta_{2}}$. 


\section{References}

Black, Fischer, and Robert Litterman, 1992, Global portfolio optimization, Financial Analysts Journal 48, 28-43.

Chavas, Jean-Paul, 2004, Risk analysis in theory and practice (Elsevier Academic Press).

Cull, Robert, Asli Demirgüç-Kunt, and Jonathan Morduch, 2007, Financial performance and outreach: A global analysis of leading microbanks, The Economic Journal 117, F107-F133.

— 2009, Microfinance meets the market, Journal of Economic Perspectives 23(1), 167-192.

D’Antonio, Louis, Tommi Johnsen, and Bruce Hutton, 2000, Socially responsible investing and asset allocation, Journal of Investing 9, 65-73.

D’Antonio, Louis, Tommi Johnsen, and R. Bruce Hutton, 1997, Expanding socially screened portfolios: An attribution analysis of bond performance, Journal of Investing 6, 79-87.

Dorfleitner, Gregor, and Michael Krapp, 2007, On multiattributive risk aversion: some claryfying results, Review of Managerial Science 1, 47-63.

Dunn, Brian, 2006, Modern portfolio theory - with a twist: The new efficient frontier, http://americansforcommunitydevelopment.org/supportingdownloads/AcquillianPresentation07.pdf.

Dupré, Denis, Isabelle Girerd-Potin, and Raghid Kassoua, 2004, Adding an ethical dimension to portfolio management, Finance India 18, 625-642.

Epstein, Larry G., and Stephen M. Tanny, 1980, Increasing generalized correlation: a definition and some economic consequences, Canadian Journal of Economics 13, 16-34.

Fabozzi, Frank J., K.C. Ma, and Becky J. Oliphant, 2008, Sin stock returns, Journal of Portfolio Management 35(1), 82-94.

Hoepner, Andreas G.F., 2007, A categorisation of the responsible investment literature, SSRN.

Markowitz, Harry M., 1952, Portfolio selection, Journal of Finance 7, 77-91.

_ 1987, Mean-Variance Analysis in Portfolio Choice and Capital Markets (Blackwell).

Morduch, Jonathan, 2000, The microfinance schism, World Development 28(4), 617-629.

Renneboog, Luc, Jenke Ter Horst, and Chendi Zhang, 2008, Socially responsible investments: Institutional aspects, performance, and investor behavior, Journal of Banking and Finance 32, 17231742.

Rhyne, Elisabeth, 2009, Microfinance for bankers and investors: Understanding the opportunities and challenges of the market at the bottom end of the pyramid (McGraw Hill).

Sen, Amartya, 1987, On Ethics and Economics. (Basil Blackwell Ltd). 
Tobin, James, 1958, Liquidity preference as behavior towards risk, Review of Economic Studies 67, $65-86$.

Yunus, Muhammad, 2007, Creating a World Without Poverty: Social Business and the Future of Capitalism (Perseus Books Group). 